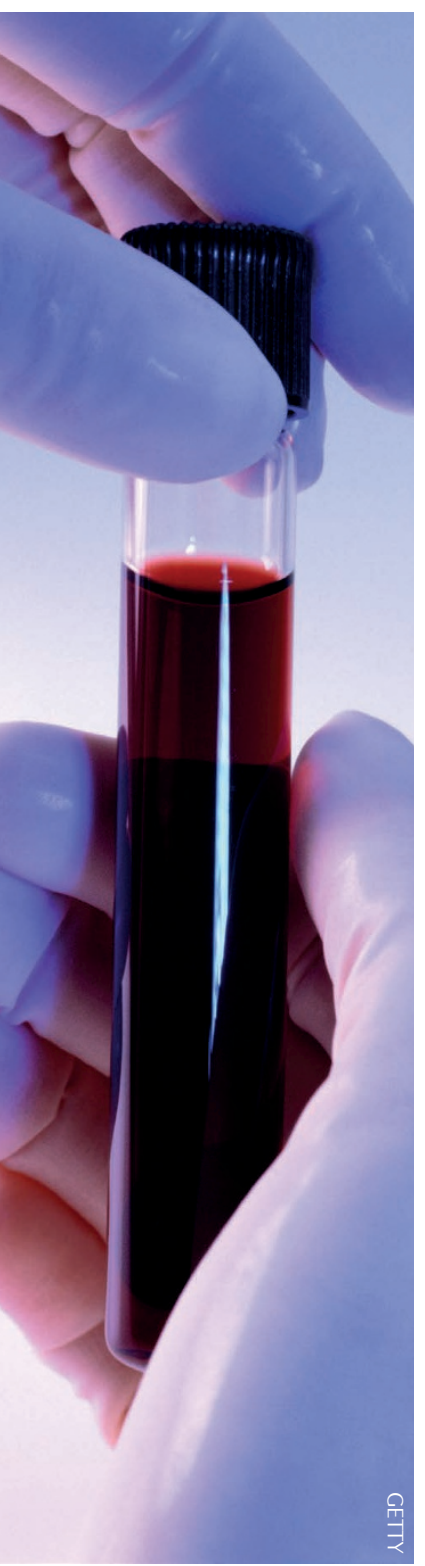

$\Rightarrow$ PROSTATE CANCER

\title{
Solo PSA test does not lower mortality
}

Use of a single PSA screening intervention does not reduce prostate cancer mortality after a median follow-up duration of 10 years, but does increase the detection of lowrisk prostate cancer, according to a new UK study.

"Previous trials that performed multiple PSA tests over time found that many men were diagnosed with low-risk, harmless cancers that were unlikely to progress or require treatment," says corresponding author Richard Martin. "These men may face unnecessary anxiety and medical investigations, as well as unnecessary treatments with side effects that can affect daily life, such as problems with erections, loss of fertility, incontinence, or bowel problems. These concerns about overdiagnosis and overtreatment of harmless disease have influenced the decisions not to introduce prostate cancer screening in many other countries."

"The Cluster Randomized Trial of PSA Testing for Prostate Cancer (CAP) offered a one-off PSA test to men aged 50-69 years in the UK," says Martin. "The goal of this lowintensity, one-off PSA testing was to avoid unnecessary screening while still identifying men with high-risk, aggressive cancers for whom screening and early detection can reduce morbidity and mortality."
The primary analysis of the CAP trial involved 408,825 men aged 50-69 years in 573 general practices in the UK. The 189,386 men in the intervention group were invited to have a one-off PSA test to detect whether they had prostate cancer. Men with PSA levels $\geq 3.0 \mathrm{ng} / \mathrm{ml}$ were then offered a standardized 10-core transrectal ultrasonography (TRUS)guided biopsy. Those diagnosed with clinically localized prostate cancer were invited to take part in the ProtecT trial, which was designed to compare surgery, radiotherapy, and active monitoring. The 219,439 men in the control group received standard National Health Service management, with information about PSA testing only being provided to men who requested it.

In the intervention group, 67,313 men underwent PSA testing and 6,857 had a PSA level between $3 \mathrm{ng} /$ $\mathrm{ml}$ and $19.9 \mathrm{ng} / \mathrm{ml}$ (making them eligible for the ProtecT trial). In total, 5,850 of these men underwent prostate biopsy. PSA testing in the control group was indirectly estimated at $10-15 \%$ over 10 years.

After a median follow-up period of 10 years, no significant difference in prostate-cancer-specific mortality was seen between the groups (549 deaths from prostate cancer in the intervention group versus 647 deaths from prostate cancer in the control group; $P=0.50$ ). The percentage of men diagnosed with prostate cancer was higher in the intervention group than in the control group $(4.3 \%$ versus $3.6 \% ; P<0.001$ ), and the intervention group also had more prostate cancer tumours with a Gleason grade $\leq 6(1.7 \%$ versus $1.1 \% ; P<0.001)$.

"Although the study did detect some dangerous cancers that could benefit from treatment, the approach of oneoff PSA testing and TRUS-guided biopsy missed a number of lethal cancers," says Martin. "The CAP trial adds to the evidence that PSA screening detects some disease that would be unlikely to cause any harm but also misses some aggressive prostate cancers that do need treatment. Overall, screening with a one-off PSA test does not save lives after an average of 10 years of follow-up after the disease is detected, but we must wait longer to see if it makes a difference at 15 years and 20 years. In addition, finding a way to improve the number of aggressive cancers that get caught in time, while reducing the number of men who have screening, biopsies, or treatment unnecessarily, remains important."

Rebecca Kelsey

ORIGINAL ARTICLE Martin, R. M. et al. Effect of a low-intensity PSA-based screening intervention on prostate cancer mortality.JAMA 319, 883-895 (2018) 